\section{ORIGINAL RESEARCH}

D. Riva

S. Bulgheroni

D. Aquino

F. Di Salle

M. Savoiardo

A. Erbetta

\title{
Basal Forebrain Involvement in Low-Functioning Autistic Children: A Voxel-Based Morphometry Study
}

BACKGROUND AND PURPOSE: Imaging studies have revealed brain abnormalities in the regions involved in functions impaired in ASD (social relations, verbal and nonverbal communication, and adaptive behavior). We performed a VBM whole-brain analysis to assess the areas involved in autistic children with DD.

MATERIALS AND METHODS: Twenty-one developmentally delayed children with ASD (aged 3-10 years) were compared with 21 controls matched for age, sex, and sociocultural background. All ASD cases had been diagnosed according to Diagnostic and Statistical Manual of Mental Disorders, Fourth Edition criteria, with the Autism Diagnostic Observation Schedule-Generic, and the Autism Diagnostic Interview-Revised. The VBM data, covaried with intelligence quotient, age, and brain volume, were analyzed.

RESULTS: ASD patients showed a pattern of regional GM reduction symmetrically affecting the basal forebrain, accumbens nucleus, cerebellar hemispheres, and perisylvian regions, including insula and putamen. Asymmetric involvement of GM was observed in other brain regions functionally connected to the basal forebrain, ie, an area located close to the medial and ventral surface of the frontal lobe. No regional WM differences were observed between the 2 groups. No significant differences between patients and controls were found regarding total brain volume, GM, and WM.

CONCLUSIONS: In children with ASD and DD, the novel finding of our VBM study was the demonstration of reduced GM volume in the basal forebrain and the areas connected with it. This system is involved in social behavior, communication, and cognitive skills. Whether the involvement of the basal forebrain is characteristic of ASD or is related to the DD present in our patients needs further investigation.

\begin{abstract}
ABBREVIATIONS: $A S D=$ autistic spectrum disorders; $B A=$ Brodmann area; $B F=$ basal forebrain $\mathrm{DD}=$ developmental delay; $\mathrm{DLPFC}=$ dorsolateral prefrontal cortex; FDR = false discovery rate; $f M R I=$ functional $M R$ imaging; $G M=$ gray matter; $I F G=$ inferior frontal gyrus; $I Q=$ intelligence quotient; $L F=$ low functioning; NACC = accumbens nucleus; $P E T$ = positron-emission tomography; $\mathrm{rCBF}=$ relative cerebral blood flow; $\mathrm{SMA}=$ supplementary motor area; $\mathrm{VBM}=$ voxel-based morphometry; $\mathrm{WM}=$ white matter
\end{abstract}

T he ASD are currently seen as genetic neurodevelopmental disorders, though their underlying biologic causes remain to be established. The general behavioral phenotype defined as ASD includes diverse endophenotypes sharing 3 core features: impaired social interaction; verbal and nonverbal communication anomalies; and restricted patterns of behavior, stereotyped patterns of behavior, or both. Furthermore, frequent comorbidities (eg, epilepsy, mental retardation, and psychiatric disorders) make it difficult to isolate the neuropathologic picture of autism. ${ }^{1}$

In patients with ASD, conventional and advanced imaging studies, such as VBM, demonstrated abnormalities in frontal lobe, temporal and parietal cortices, language areas, amygdala, caudate nucleus, and cerebellum, even though some findings were controversial. ${ }^{1}$ Furthermore, most studies were carried out in high-functioning patients or patients with Asperger

Received October 12, 2010; accepted after revision December 17.

From the Division of Developmental Neurology (D.R., S.B.) and Department of Neuroradiology (D.A., M.S., A.E.), Fondazione IRCCS Istituto Neurologico C. Besta, Milan, Italy; and Department of Cognitive Neuroscience (F.D.S.), University of Maastricht, Maastricht, the Netherlands.

Please address correspondence to Daria Riva, MD, Developmental Neurology Division, Fondazione IRCCS Istituto Neurologico C. Besta, Via Celoria, 11-0133 Milan, Italy; e-mail: driva@istituto-besta.it

http://dx.doi.org/10.3174/ajnr.A2527 syndrome. However, most of the autistic population is intellectually impaired, and $\approx 80 \%$ of autistic children are LF, ie, they have an IQ $<70 .^{2}$ Despite this prevalence of LF children with ASD, these patients are rarely investigated, ${ }^{3-5}$ probably because of the difficulties in cooperating for neuroradiologic investigations.

The aim of our study was to investigate LF children with ASD younger than age 10 with VBM, which is an unbiased, operator-independent method for detecting statistically significant differences between groups. ${ }^{6}$

\section{Materials and Methods}

\section{Subjects}

From 93 children with ASD, referred to our Developmental Neurology Unit from January 2006 to August 2008, a group of 21 LF children with idiopathic ASD without associated seizures or other neurologic diseases was selected. Patients with known infectious, metabolic, or genetic diseases, and chromosomal abnormalities were excluded. Children with disintegrative disorder also were excluded. In addition, we excluded patients in whom conventional MR imaging demonstrated structural abnormalities. Four patients were excluded because of motion artifacts.

Of the 21 patients ( 8 females and 13 males; age range, 3 years and 2 months-10 years and 10 months; mean, 6 years and 6 months; SD, 


\begin{tabular}{|c|c|c|c|c|c|}
\hline & \multicolumn{2}{|c|}{ ASD Group } & \multicolumn{2}{|c|}{ Control Group } & \multirow{2}{*}{$\begin{array}{c}t \text { Test, } \\
P \text { Value }\end{array}$} \\
\hline & Mean (SD) & Range & Mean (SD) & Range & \\
\hline Age (mo) & $78(29)$ & $38-130$ & $82(25)$ & $45-123$ & 0.623 \\
\hline Total volume of GM (ml) & $926.06(230)$ & 239.34-1485.22 & $925.50(107.43)$ & 775.89-1176.89 & 0.992 \\
\hline Total volume of WM (ml) & $460.35(249.77)$ & 312.34-1439.69 & 391.33 (46.92) & $323.69-478.83$ & 0.227 \\
\hline Total volume of brain (ml) & $1684.86(480.07)$ & 1185.71-3000.20 & $1597.04(320.66)$ & 1231.94-2263.61 & 0.490 \\
\hline Total volume of CSF (ml) & $298.44(274.46)$ & 104.36-1321.17 & $280.20(218.19)$ & 111.11-793.96 & 0.813 \\
\hline
\end{tabular}

2 years and 5 months), 12 had a diagnosis of autism, and 9 of Pervasive Developmental Disorder-Not Otherwise Specified. In all cases, the diagnosis was established according to the Diagnostic and Statistical Manual of Mental Disorders, Fourth Edition diagnostic criteria and confirmed by semistructured assessment by using Autism Diagnostic Observation Schedule-Generic and by interviewing the parents by using Autism Diagnostic Interview-Revised.

The cognitive functioning was assessed by using the Wechsler Intelligence Scale according to their respective age, the Leiter International Performance Scale Revised for nonverbal children, and the Griffiths Mental Developmental Scale for those with chronologic or mental age $<4$ years. The IQ ranged from 32 to 66 (mean, $52.5 \pm 9.8$ ).

The control group consisted of 21 normally developing children ( 8 females and 13 males; age range, 3 years and 9 months- 10 years and 3 months; mean, 6 years and 10 months; SD, 2 years and 1 month), with normal IQ, no signs of autistic behavior or other psychiatric or neurologic disorders, or family history of neurologic or psychiatric illness among their first-degree relatives. The study was approved by our local ethics committee, and all examinations were performed with the written informed consent of the patients' parents.

\section{MR Imaging Acquisition}

All the autistic patients were examined under propofol sedation (1 $\mathrm{mg} / \mathrm{kg}$ ). The controls $<6$ years old also were examined under propofol sedation. They were recruited among inpatients with suspected spinal cord abnormalities and were included in the study if the brain and spine examination was normal. The controls $>6$ years old were examined without sedation; they were recruited among the children of the medical and technical staff involved in the study. Volumetric T1-weighted images were acquired on a 1.5T MR imaging system (Siemens, Erlangen, Germany) by using a magnetization-prepared gradient-echo sequence $(\mathrm{TR}=1640 \mathrm{~ms}, \mathrm{TE}=2.48 \mathrm{~ms}, \mathrm{TI}=552 \mathrm{~ms}$, $\mathrm{FOV}=256 \times 256 \mathrm{~mm}$, matrix $=256 \times 256,160$ sagittal sections, voxel size $=1 \times 1 \times 1 \mathrm{~mm}$ ).

Structural imaging included axial proton-density/T2-weighted images $(\mathrm{TR}=3500 \mathrm{~ms}, \mathrm{TE}=17 \mathrm{~ms} / 84 \mathrm{~ms}, \mathrm{FOV}=208 \times 256 \mathrm{~mm}$, matrix $=208 \times 256$, section thickness $=5 \mathrm{~mm}$ ) and coronal turbo spin-echo T2-weighted images $(\mathrm{TR}=4100 \mathrm{~ms}, \mathrm{TE}=143 \mathrm{~ms}$, FOV $=$ $324 \times 384 \mathrm{~mm}$, matrix $=324 \times 384$, section thickness $=5 \mathrm{~mm})$. Structural imaging was assessed by a senior neuroradiologist for the presence or absence of supratentorial and infratentorial abnormalities and signal intensity changes, based on visual inspection. The MR imaging obtained for the controls was similarly reviewed.

\section{VBM Analysis}

Images were processed according to the optimized VBM method implemented in SPM5 (http://www.fil.ion.ucl.ac.uk/spm/software/ spm5/) as described previously. ${ }^{7}$ Original T1 images were first normalized to a pediatric (5-9.5 years) brain template (Cincinnati Children's Hospital Medical Center: CCHMC2_y; available at https:// irc.cchmc.org) by using a 12-parameter linear affine transformation and a nonlinear transformation through $7 \times 8 \times 7$ nonlinear basis functions and then segmented into GM, WM, and CSF partitions. A custom template was then obtained as suggested by Good et al, ${ }^{7}$ normalizing, segmenting, smoothing, and averaging the images of the 42 subjects of our study to account for potential differences between our MR scanner and the scanner used to obtain the pediatric template and to account for differences between our sample and the sample used for the template. The original, native $\mathrm{T} 1$ images were then normalized and segmented again by using the new template and priors. To preserve the amount of a particular tissue (GM) within a voxel, a further modulation step was incorporated.

Finally, the images were smoothed with a Gaussian kernel filter of $8 \times 8 \times 8 \mathrm{~mm}$ full width half maximum to have data more normally distributed and to compensate for inexact spatial normalization.

Differences in GM and WM were assessed statistically using the general linear model based on the Gaussian field theory. An analysis of covariance, with total intracranial volume, age, IQ, and sex as covariates, was used. The significance levels were set at $P<.05$ corrected for multiple comparisons by using the FDR.

Localization of areas of significant tissue loss was determined by superimposing the regions of significant atrophy on the averaged T1weighted image used to create the template. Regions of interest were reported in Montreal Neurologic Institute coordinates.

\section{Total Volumes Analysis}

The intracranial volumes were estimated by integrating the voxel values across the modulated (unsmoothed) GM, WM, and CSF partitions, and results were converted from voxels into milliliters. Group differences in total intracranial volume as well as group differences in total GM, WM, and CSF volumes were assessed by using a 2-tailed $t$ test.

\section{Results}

\section{Quantitative Structural MR Imaging}

No significant differences were found between cases and controls regarding the total volumes of brain $(P=.49), \mathrm{GM}(P=$ $.992)$, WM $(P=.227)$, and CSF $(P=.813)$. These results are summarized in Table 1.

\section{Regional WM Differences}

No regional differences in the WM were observed between the 2 groups.

\section{Regional GM Differences}

The autistic patients had a reduced volume of GM in 13 3D clusters (significance threshold, $P<.05$, FDR corrected). Basal forebrain, nucleus accumbens, SMA, cerebellum, and perisylvian region (including the insula and putamen) showed 
Table 2: GM regions significantly reduced $(P<.05$ FDR corrected) in the autistic group by comparison with the controls

\begin{tabular}{|c|c|c|c|}
\hline Anatomic Location & $\begin{array}{l}\text { Talairach Coordinates } \\
\text { Center of Cluster, } x \text { y z }\end{array}$ & $\begin{array}{c}\text { Peak of Significance } \\
\text { ( } t \text { score })\end{array}$ & $\begin{array}{l}\text { Cluster size } \\
\text { (No. of voxels) }\end{array}$ \\
\hline Basal forebrain, nucleus accumbens & $0 \quad 1-2$ & 4.05 & 1282 \\
\hline Supplementary motor area (BA6) & $2-1 \quad 47$ & 4.12 & 699 \\
\hline Left insula, left putamen, left perisylvian cortex (BA48) & $\begin{array}{lll}-43 & -2 & 13\end{array}$ & 5.37 & 6945 \\
\hline Right insula, right putamen, right perisylvian cortex (BA48) & $34-2 \quad 14$ & 5.42 & 8266 \\
\hline Left cerebellum & $-26-58-37$ & 5.56 & 6452 \\
\hline Right cerebellum & $23-55-39$ & 5.29 & 10,441 \\
\hline Left DLPFC (BA47) & $-44 \quad 15 \quad 34$ & 4.93 & 1513 \\
\hline Left inferior temporal gyrus (BA20) & $-46-40-14$ & 5.40 & 1708 \\
\hline Left middle temporal gyrus (BA21) and left superior temporal gyrus (BA22) & $-56-19-2$ & 5.96 & 2844 \\
\hline Left precuneus (BA7) & $-1-68 \quad 34$ & 5.78 & 1225 \\
\hline Left occipito-basal cortex (BA18) & $-14-78-18$ & 5.48 & 359 \\
\hline Left inferior frontal gyrus (BA44) & $\begin{array}{lll}-39 & 22 & 2\end{array}$ & 5.39 & 276 \\
\hline Right postcentral cortex $(\mathrm{BA} 1,2,3)$, right inferior parietal lobe $(\mathrm{BA} 40)$ & $41-33 \quad 40$ & 5.35 & 1382 \\
\hline
\end{tabular}

Note:-All labels are derived from the Anatomical Automatic Atlas. The $x, y, z$ coordinates are the Talairach coordinates of the center of each $3 \mathrm{D}$ cluster. ${ }^{43}$ BAs also are provided.

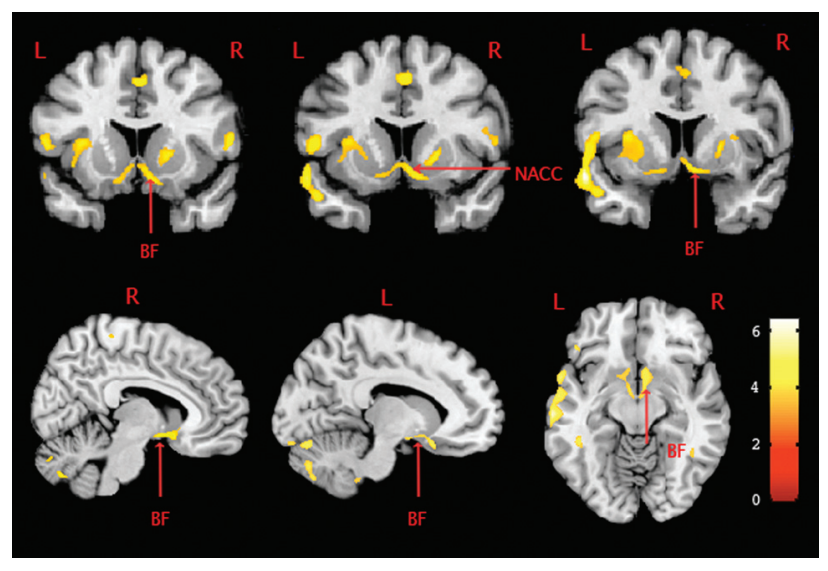

Fig 1. Basal forebrain and accumbens nucleus volume differences between groups. GM volume differences between groups, overlaid on T1 images (consecutive coronal sections, rostral to caudal) showing the involvement of accumbens nucleus and basal forebrain. The relative decrease in GM attenuation observed in autistic subjects compared with controls is represented in a red-orange color scale. The statistical threshold is $P<.05$ (FDR corrected). $L=$ left; $R=$ right.

a reduced GM bilaterally. In the left hemisphere, a reduction in GM was recorded in the occipital basal cortex; inferior, middle, and superior temporal gyri; IFG; DLPFC; and precuneus. In the right hemisphere, a GM reduction was visible in the postcentral cortex and inferior parietal lobe. These results are shown in Table 2 and Figs 1 and 2.

\section{Discussion}

In this study we applied unbiased whole brain VBM to assess neuroanatomic changes in LF autistic children compared with controls paired for age, sex, and social status. The choice of normal IQ for the control group was dictated by the fact that a group of DD children with IQ matching with that of the patients would probably be a heterogeneous sample of various pathologies that would create even greater difficulties in the comparison.

Several VBM investigations in the autistic population have been reported in the literature. These studies demonstrated a volume loss in cortical and subcortical areas as well as in the cerebellum. The affected areas were the IFG, ${ }^{8,9}$ insula, ${ }^{9}$ cerebellum, ${ }^{5,10}$ precuneus, ${ }^{11}$ and basal ganglia. ${ }^{11,12}$ These areas also have been found affected in our study. Higher than normal density, not observed by us, has been reported in inferior

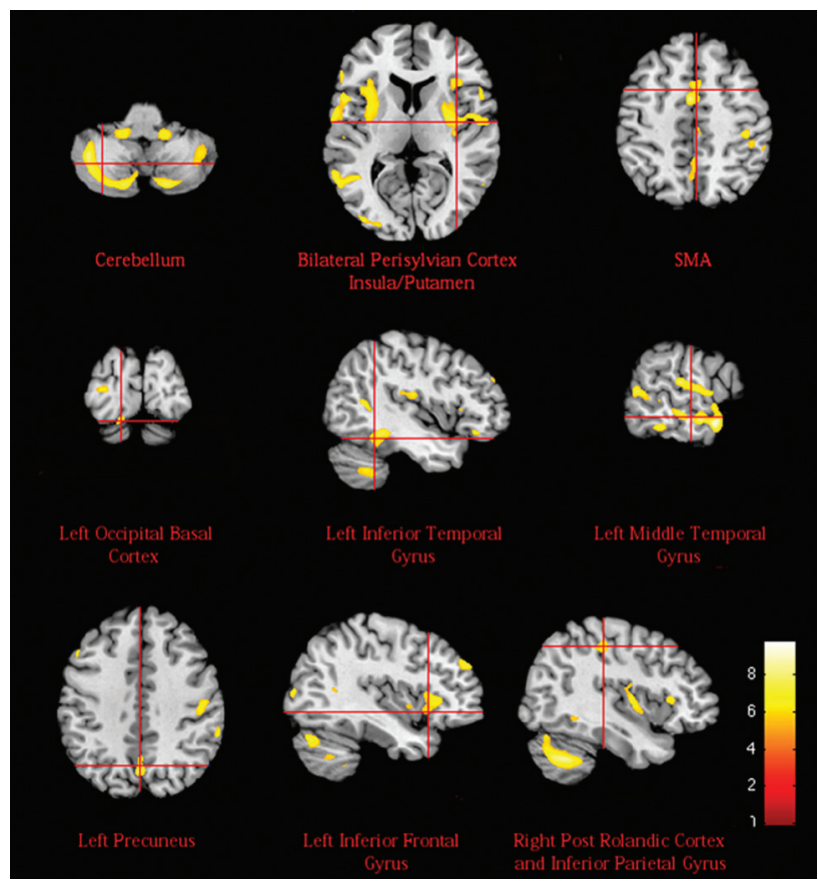

Fig 2. GM volume differences between groups. GM volume differences between groups, overlaid on $\mathrm{T} 1$ images. The relative decrease in GM attenuation observed in autistic subjects compared with controls is represented in a red-orange color scale. The statistical threshold is $P<.05$ (FDR corrected).

frontal cortex ${ }^{10,13}$ and cerebellum. ${ }^{3,8,14}$ Other investigations identified areas of higher or lower GM density that were unaffected in our patients, such as the amygdala, ${ }^{8}$ cingulate gyrus, ${ }^{8,11}$ fusiform gyrus, ${ }^{10,14}$ and thalamus. ${ }^{5,12,13}$ GM reduction in the basal forebrain, which is the most remarkable finding of our study, was not observed previously.

In our series of ASD patients, volume loss was found to affect the basal forebrain, nucleus accumbens, perisylvian regions, and other cortical brain regions functionally connected to the abovementioned areas as well as the putamen and cerebellum.

The basal forebrain comprises a group of structures located in the medial and ventral surface of the frontal lobe. The basal forebrain consists of 3 functionally distinct compartments, ${ }^{15}$ ie, the mainly cholinergic corticopetal system, the extended amygdalae, and the ventral striatopallidal system. The cholin- 
ergic cells are concentrated in the septum, the diagonal band, and the nucleus basalis of Meynert ${ }^{16}$; their medial component projects to the medial frontal and cingulate cortex, whereas the more lateral subdivisions of this system project to the lateral neocortex, including the orbitoinsular, prefrontal, temporal, parietal, and occipital cortices. ${ }^{16,17}$

Therefore, the term basal forebrain commonly refers to an extended continuum of subcortical neurons providing projections to a variety of neocortical fields and limbic structures implicated in many cognitive functions and social behavior patterns. ${ }^{18}$ The basal forebrain activates areas of the cerebral cortex and facilitates learning, by using acetylcholine as a prominent neurotransmitter. Functionally, the cholinergic system has been referred to as an "action system" that helps individuals to develop the ability to focus on the environment and provide a coherent behavioral response. ${ }^{19}$

Volume loss in the basal forebrain is consistent with the hypothesis proposed by Perry et al, ${ }^{20}$ according to which, in autism, the cholinergic system of the basal forebrain is impaired. In fact, in autistic subjects, they demonstrated a lower level of cholinergic receptors than in normal controls. Cholinergic afferents innervate the cerebral cortex during the early postnatal development, which is the most dynamic period of neuronal differentiation and synapse formation. ${ }^{21}$ A disruption of the cholinergic innervation results in a delayed cortical neuronal development and permanent changes in cortical cytoarchitecture and cognitive functions. ${ }^{21}$

Involvement of the basal forebrain has been demonstrated by imaging studies in attention, learning, memory, and reward. In an auditory vigilance test, PET showed $\mathrm{rCBF}$ changes in the basal forebrain. ${ }^{22}$ During classic conditioning tasks with auditory discrimination, PET showed that rCBF of the auditory cortex positively correlated with activity in the basal forebrain, amygdala, and orbitofrontal cortex. ${ }^{23}$ These results support the notion that the basal forebrain's cholinergic projection to the cortex is important in modulating the synaptic activity. Furthermore, PET studies suggested an activity in the nucleus of the diagonal band of Broca in episodic memory recall. ${ }^{24}$ The basal forebrain and DLPFC were activated in paradigms sensitive to working memory. ${ }^{25}$

The extended amygdalae stretch medially and rostrally from the centromedial nuclei of the amygdalae through the substantia innominata into the medial portion of the nucleus accumbens, thus joining the ventral striatopallidal system. In our study, the nucleus accumbens was found to be reduced in volume. This nucleus has a central role in the functional interplay between basal forebrain components, and between the basal forebrain and other brain regions. It receives projections from the hippocampus; basolateral amygdalae; and the prefrontal, insular, and temporal association cortex, ${ }^{15}$ and it has efferent connections to the corticopetal cholinergic system, thus modulating the cholinergic output. ${ }^{15,16}$ The nucleus accumbens is therefore involved in memory; it is engaged both in incentive-reward processes and in adaptive responses to conditioned and unconditioned aversive stimuli. A reduced volume of the nucleus accumbens also was reported by Ballmaier et $\mathrm{al}^{26}$ in children with schizophrenia. In their study, the smaller left nucleus accumbens was associated with linguistic errors and the smaller right nucleus accumbens with difficulties in planning and organized thinking.
A reduced volume of GM was found in the SMA (BA6) and putamen, structures involved in the motor and cognitive circuits. SMA is functionally subdivided in the SMA proper and pre-SMA. Functional imaging studies in humans have shown that the pre-SMA has an important role in supramotor activities. ${ }^{27}$ Similarly, the putamen is activated both when a subject is preparing to move and when the movement is performed. Motor symptoms, such as postural abnormalities in the head and trunk, and stereotypical movements are prominent features of autism; they are probably due to involvement of both the fronto-striatal basal ganglia circuits and the cerebellum. ${ }^{28}$

A reduced volume of GM was identified bilaterally in the insula and the perisylvian region. The insular cortex works as a relay area for multiple neurocognitive systems and plays a part in many functions related to emotions or regulating the body's homeostasis. This region may therefore be involved in the complex clinical features of autistic disorders. ${ }^{9}$ The anterior cortical perisylvian areas are important for speech and language functions.

Our patients showed GM reduction in both cerebellar hemispheres. The cholinergic basal forebrain is connected with the cerebellum ${ }^{29}$ and the cholinergic cerebellar neurons contribute to mental functions related to cognition, behavior and emotions. In autism, there are numerous reports of volume changes in the cerebellar vermis. Individuals with early infantile autism show fourth ventricle enlargement, loss of Purkinje cells in the lateral and inferior cerebellar cortex, and abnormal or reduced numbers of neurons in the deep cerebellar nuclei. ${ }^{1}$

Unilateral involvement was found in different areas of the cerebral hemispheres and was more numerous on the left side.

GM loss was found in the left DLPFC. This area is part of the neuronal network underlying social cognition in the frame of the theory of mind, and executive functions. GM loss in the frontal lobe of autistic children was confirmed by imaging and postmortem studies. ${ }^{1}$ Decreased connections between the frontal cortex and regions implicated in social cognition and in the empathic processes were demonstrated by diffusion tensor imaging. ${ }^{30}$

Reduced GM was prominent in the left middle temporal gyrus, which is functionally connected to the cholinergic system and nucleus accumbens. ${ }^{17}$ The middle temporal cortex and superior temporal gyrus are important for perceiving and decoding other people's gaze, a function deficient in autistic children. An impairment of this region also could contribute to the language difficulties and consequent communication problems of autistic subjects. ${ }^{31}$

GM loss in our ASD patients involved the precuneus that is connected with multiple areas of the interhemispheric cortex, convexity, basal ganglia, and thalami. ${ }^{32}$ Changes occurring in this pivotal area are consistent with recent claims that autism is a connectivity disorder. ${ }^{33}$ The precuneus is activated when we make judgments that demand empathy and when we attribute emotions to ourselves and to other people. ${ }^{34}$

A reduction in GM was recorded in the left inferior temporal gyrus and occipito-basal region: there is a growing body of literature suggesting that individuals with autism, pervasive developmental disorder, and Asperger syndrome have abnormalities in perceiving faces and objects. Together with the parahippocampal and lateral occipital gyri, the inferior tem- 
poral gyrus seems to be involved in common object perception. In autistic subjects, Schultz et $\mathrm{al}^{35}$ found fMRI abnormalities in the inferior temporal and fusiform gyri in face discrimination tasks.

Involvement of the left IFG deserves a specific comment. This region, the inferior parietal lobe and BA1, 2, 3 are part of the mirror neuron system implicated in understanding other people's actions, intentions, and emotions. ${ }^{36}$ In autistic patients, a limited activation of the area containing the mirror neurons was demonstrated by fMRI. ${ }^{37}$ In our study, these 2 last areas, inferior parietal lobe and BA1, 2, 3 were found affected only on the right side.

In contrast with the studies collected by Radua et $\mathrm{al}^{38}$ in agreement with other studies, ${ }^{9,10,13}$ we did not find any volume abnormalities in the WM. These different results can be attributed to the heterogeneity of the disorder and the different age of the population studied. Diffusion tensor imaging studies might be more sensitive to WM abnormalities.

Bilateral GM reduction could be consistent with more prominent behavioral abnormalities than unilateral GM involvement, as already shown in bilateral versus unilateral focal brain lesions. ${ }^{39}$ However, only a clinical correlation could confirm this hypothesis.

Abnormalities in the basal forebrain were reported in other syndromes with DD, such as Down syndrome, ${ }^{40}$ Rett syndrome, ${ }^{41}$ and fragile $\mathrm{X}$ syndrome, ${ }^{42}$ but not in children with idiopathic DD. ${ }^{42}$

The autistic phenotype we examined cannot be merely summarized in the term autistic behavior plus intellectual disability, because it comprises a syndromic constellation of symptoms, in which there are both signs of autism and of cognitive malfunctioning. Involvement of the basal forebrain, however, might be an indicator of DD rather than autism itself.

The limitations of our study are the small sample size and the lack of a control group with idiopathic DD. The analysis of such a group could answer the question whether basal forebrain involvement is related to the mental retardation rather than autism itself.

\section{Conclusions}

The main finding of this study was the volume reduction in the basal forebrain, an area not previously found affected in VBM studies of autistic children. This area and its extensive, multiple cortical connections that also present volume reduction, are involved in social, communicative, and cognitive behavior. The basal forebrain involvement might be related to DD, which was always present in our autistic children.

\section{Acknowledgments}

We thank all the children who participated in the study and those who acted as the healthy controls and their families. We also thank Dagmar Di Fiore, RT, for helpfulness and accuracy in performing the MR examinations as well as the reviewers for many helpful suggestions.

\section{References}

1. Amaral DG, Schumann CM, Nordahl CW. Neuroanatomy of autism. Trends Neurosci 2008;31:137-45
2. Fombonne E. Epidemiology of pervasive developmental disorders. Pediatr Res 2009;65:591-98

3. Bonilha L, Cendes F, Rorden C, et al. Gray and white matter imbalancetypical structural abnormality underlying classic autism? Brain Dev 2008;30:396-401

4. Boddaert N, Chabane N, Gervais $\mathrm{H}$, et al. Superior temporal sulcus: anatomical abnormalities in childhood autism: a voxel-based morphometry MRI study. Neuroimage 2004;23:364-69

5. Spencer MD, Moorhead TW, Lymer GK, et al. Structural correlates of intellectual impairment and autistic features in adolescents. Neuroimage 2006;33:1136-44

6. Ashburner J, Friston KJ. Voxel-based morphometry: the methods. Neuroimage 2000;11:805-21

7. Good CD, Johnsrude IS, Ashburner J, et al. A voxel-based morphometric study of ageing in 465 normal adult human brains. Neuroimage 2001;14:21-36

8. Abell F, Krams M, Ashburmer J, et al. The neuroanatomy of autism: a voxel based whole brain analysis of structural scans. Neuroreport 1999;10:1647-51

9. Kosaka H, Omori M, Munesue T, et al. Smaller insula and inferior frontal volumes in young adults with pervasive developmental disorders. Neuroimage 2010;50:1357-63

10. Rojas DC, Peterson E, Winterrowd E, et al. Regional gray matter volumetric changes in autism associated with social and repetitive behavior symptoms. BMC Psychiatry 2006;6:56-68

11. McAlonan GM, Cheung V, Cheung C, et al. Mapping the brain in autism. A voxel-based MRI study of volumetric differences and intercorrelations in autism. Brain 2005;128:268-76

12. McAlonan GM, Suckling J, Wong N, et al. Distinct patterns of grey matter abnormality in high-functioning autism and Asperger's syndrome. J Child Psychol Psychiatry 2008;49:1287-95

13. Waiter GD, Williams JH, Murray AD, et al. A voxel-based investigation of brain structure in male adolescents with autistic spectrum disorder. Neuroimage 2004;22:619-25

14. Ke X, Hong S, Tang T, et al. Voxel-based morphometry study on brain structure in children with high-functioning autism. Neuroreport 2008;19:921-25

15. Zahm DS. The evolving theory of basal forebrain functional-anatomical macrosystems. Neurosci Biobehav Rev 2006;30:148-72

16. Selden NR, Gitelman DR, Salamon-Murayama N, et al. Trajectories of cholinergic pathways within the cerebral hemispheres of the human brain. Brain 2008;121:2249-57

17. Zaborszky L, Hoemke L, Mohlberg H, et al. Stereotaxic probabilistic maps of the magnocellular cell groups in human basal forebrain. Neuroimage 2008;42:1127-41

18. Baxter MG, Chiba AA. Cognitive functions of the basal forebrain. Curr Opin Neurobiol 1999;9:178-83

19. Lam KS, Aman MG, Arnold LE. Neurochemical correlates of autistic disorder: a review of the literature. Res Dev Disabil 2006;27:254-89

20. Perry EK, Lee ML, Martin-Ruiz CM, et al. Cholinergic activity in autism: abnormalities in the cerebral cortex and basal forebrain. Am J Psychiatry 2001;158:1058-66

21. Hohmann CF, Berger-Sweeney J. Cholinergic regulation of cortical development and plasticity. New twists to an old story. Perspect Dev Neurobiol 1998;5:401-25

22. Paus T, Perry DW, Zatorre RJ, et al. Modulation of cerebral blood flow in the human auditory cortex during speech: role of motor-to-sensory discharges. Eur J Neurosci 1996;8:2236-46

23. Morris JS, Friston KJ, Dolan RJ. Experience-dependent modulation of tonotopic neural responses in human auditory cortex. Proc Biol Sci 1998;265:649-57

24. Fujii T, Okuda J, Tsukiura T, et al. The role of the basal forebrain in episodic memory retrieval: a positron emission tomography study. Neuroimage 2002;15:501-08

25. Swartz BE, Halgren E, Fuster JM, et al. Cortical metabolic activation in humans during a visual memory task. Cereb Cortex 1995;5:205-14

26. Ballmaier M, Toga AW, Siddarth $\mathrm{P}$, et al. Thought disorder and nucleus accumbens in childhood: a structural MRI study. Psychiatry Res 2004;130:43-55

27. Sakai K, Hikosaka O, Miyauchi S, et al. Presupplementary motor area activation during sequence learning reflects visuo-motor association. J Neurosci 1999;19:RC1:1-6

28. Rinehart NJ, Tonge BJ, Iansek R, et al. Gait function in newly diagnosed children with autism: cerebellar and basal ganglia related motor disorder. Dev Med Child Neurol 2006;48:819-24

29. Walker BR, Diefenbach KS, Parikh TN. Inhibition within the nucleus tractus solitarius (NTS) ameliorates environmental exploration deficits due to cerebellum lesions in an animal model for autism. Behav Brain Res 2007;176:109-20

30. Noriuchi M, Kikuchi Y, Yoshiura T, et al. Altered white matter fractional anisotropy and social impairment in children with autism spectrum disorder. Brain Res 2010;1362:141-49

31. Boddaert N, Belin P, Chabane N, et al. Perception of complex sounds: abnormal pattern of cortical activation in autism. Am J Psychiatry 2003;160:2057-60 
32. Cavanna AE, Trimble MR. The precuneus: a review of its functional anatomy and behavioural correlates. Brain 2006;129:564-83

33. Kennedy DP, Courchesne E. The intrinsic functional organization of the brain is altered in autism. Neuroimage 2008;39:1877-85

34. Siegal M, Varley R. Neural systems in theory of mind. Nat Rev Neurosci 2002;3:463-71

35. Schultz RT, Gauthier I, Klin A, et al. Abnormal ventral temporal cortical activity during face discrimination among individuals with autism and Asperger syndrome. Arch Gen Psychiatry 2000;57:331-40

36. Rizzolatti G, Sinigaglia C. The functional role of the parieto-frontal mirror circuit: interpretations and misinterpretations. Nat Rev Neurosci 2010;11:264-74

37. Dapretto M, Davies MS, Pfeifer JH, et al. Understanding emotions in others: mirror neuron dysfunction in children with autism spectrum disorders. Nat Neurosci 2006;9:28-30
38. Radua J, Via E, Catani M, et al. Voxel-based meta-analysis of regional whitematter volume differences in autism spectrum disorder versus healthy controls. Psychol Med 2011;41:1539-50

39. Ballantyne AO, Spilkin AM, Hesselink J, et al. Plasticity in the developing brain: intellectual, language and academic functions in children with ischaemic perinatal stroke. Brain 2008;131:2975-85

40. Chen Y, Dyakin VV, Branch CA, et al. In vivo MRI identifies cholinergic circuitry deficits in a Down syndrome model. Neurobiol Aging 2009;30:1453-65

41. Riikonen R. Neurotrophic factors in the pathogenesis of Rett syndrome. J Child Neurol 2003;18:693-97

42. Hoeft F, Carter JC, Lightbody AA, et al. Region-specific alterations in brain development in one- to three-year-old boys with fragile $\mathrm{X}$ syndrome. Proc Nat Acad Sci U S A 2010;107:9335-39

43. Talairach J, Tournox P. Co-Planar Stereotaxic Atlas of the Human Brain. New York: Thieme Medical Publishers; 1988 